\title{
Generating Realistic Smart Grid Communication Topologies Based on Real-Data
}

\author{
Thomas Hartmann*, Francois Fouquet*, Jacques Klein*, and Yves Le Traon*, \\ Alexander Pelov ${ }^{\dagger}$, Laurent Toutain ${ }^{\dagger}$, and Tanguy Ropitault ${ }^{\dagger}$ \\ *Interdisciplinary Centre for Security, Reliability and Trust (SnT), University of Luxembourg, Luxembourg, \{first.last\}@uni.lu \\ ${ }^{\dagger}$ Telecom Bretagne, France, \{first.last $\} @$ telecom-bretagne.eu
}

\begin{abstract}
Today's electricity grid must undergo substantial changes in order to keep pace with the rising demand for energy. The vision of the smart grid aims to increase the efficiency and reliability of today's electricity grid, e.g. by integrating renewable energies and distributed micro-generations. The backbone of this effort is the facilitation of information and communication technologies to allow two-way communication and an automated control of devices. The underlying communication topology is essential for the smart grid and is what enables the smart grid to be smart. Analyzing, simulating, designing, and comparing smart grid infrastructures but also optimizing routing algorithms, and predicating impacts of failures, all of this relies on deep knowledge of a smart grids communication topology. However, since smart grids are still in a research and test phase, it is very difficult to get access to real-world topology data. In this paper we provide a comprehensive analysis of the power-line communication topology of a real-world smart grid, the one currently deployed and tested in Luxembourg. Building on the results of this analysis we implement a generator to automatically create random but realistic smart grid communication topologies. These can be used by researchers and industrial professionals to analyze, simulate, design, compare, and improve smart grid infrastructures.
\end{abstract}

\section{INTRODUCTION}

Today's electricity grid was designed for the demand of the 20th century. In order to keep pace with the rising demand for energy it must undergo substantial changes. The vision of the smart grid therefore aims to increase efficiency and reliability of the electricity grid by transforming today's centralized electricity grid into a distributed, self-adaptive, and self-healing smart grid of tomorrow. In future, renewable energies and distributed micro-generations are expected to be seamlessly integrated into the electricity grid. To enable all of this, the smart grid emerges as a convergence of information and communication technologies (ICT) with power system engineering [1]. The backbone of this effort is the facilitation of modern ICT to allow two-way communication and an automated control of devices. The underlying communication topology is essential for the smart grid and is what enables the smart grid to be smart. In fact, the underlying communication topology allows most of the smart grid features, such as remote meter reading (automatically collecting consumption and status data from smart meters), remote control of devices, or demand time pricing [2]. Analyzing, simulating, designing, and comparing smart grid infrastructures but also optimizing

\footnotetext{
The research leading to this publication is supported by the National Research Fund Luxembourg (grant 6816126) and Creos Luxembourg S.A. under the SnT-Creos partnership program.
}

routing algorithms, and predicating impacts of failures, all of this relies on the knowledge of a smart grids communication topology. However, given the fact that smart grids are emerging infrastructures that are still in a research and test phase, it is usually very difficult to get access to real-world smart grid communication topologies. Therefore, many researchers as well as industrial professionals face the challenge to create their own communication topology models. Since models offer a simpler, safer and cheaper [3] means to reason, it is a common approach to build abstractions of the topology, e.g. to test smart grid architectures or build simulation tools [4]. Due to a lack of publicly available descriptions of real-world smart gird communication topologies, many researchers and industrial professionals work with general statistical graph properties rather than real-world data [4] or use theoretical graph models as topologies [5]. However, these are insufficiently capable of reflecting the characteristics of real smart grid topologies.

In this paper we provide a comprehensive analysis of the communication topology of a real-world smart grid, the one currently deployed and tested in Luxembourg. We work together with our industrial partner Creos Luxembourg S.A. ${ }^{1}$, the main electricity grid operator in Luxembourg. Creos has the role of a transmission system operator (TSO) managing the high-voltage (HV) grid but also the role of a distribution system operator (DSO) managing the medium-voltage (MV) and low-voltage (LV) grid. In this work we focus on the analysis of the communication topology rather than on electrical properties. The outcomes of our analysis point out important characteristics, restrictions, and possibilities of realworld smart grid communication topologies. The communication topology in Luxembourg is built on a power-line communication (PLC) [6] network. Therefore, the results of this work mainly apply to PLC networks. The outcomes of our analysis are typical characteristics, not just for Luxembourg, but also for PLC based smart grids in other countries. In addition to static characteristics we also provide an analysis of topology changes over time. Drawing upon these findings, we implement a generator to automatically create random but realistic smart grid communication topologies in a variety of sizes and with configurable properties. All generated topologies comply with the characteristics revealed by our analysis. They can be used to analyze, simulate, design, compare, and finally improve smart grid infrastructures

\footnotetext{
${ }^{1}$ Creos Luxembourg S.A. is the main electricity grid operator in Luxembourg. The SnT centre of the University of Luxembourg works together with Creos Luxembourg S.A. on cyber security for smart grids.
} 
The remainder of this paper is as follows. In section II we analyze and discuss the characteristics of the PLC smart grid communication topology in Luxembourg. Section III describes the implementation of our topology generator. We discuss the related work in section IV and future work in section $\mathrm{V}$. The conclusion of the paper is presented in section VI.

\section{COMmunication TOPOLOGY OF THE LUXEMBOURG SMART GRID}

In this section we analyze and describe the structure, characteristics, and evolution (over time) of the smart grid communication topology in Luxembourg. The findings are used in section III to generate random but realistic topologies.

\section{A. Topology Structure}

The smart grid communication topology in Luxembourg is built upon a PLC network. A major advantage of PLC is that the same media that is used for electric power transmission can be reused for establishing the communication network and transmitting data. On the other hand, a major concern with PLC is the amount of electrical noise and disturbances that may be encountered, which requires advanced error detection techniques. Currently, three different PLC protocols are tested in Luxembourg to connect the smart grid devices: PLC PRIME [7], PLC G3 [8], and PLC G1 [9]. The main smart grid devices forming the communication topology are:

Smart meters are the cornerstones of the new smart grid infrastructure. They are installed at customers' houses to continuously measure electric consumption and the quality of power supply and regularly report these values to utilities for monitoring and billing purposes. Initially their main task was essentially automated meter reading (AMR) [1] but nowadays they become more and more interconnected (e.g. control other devices like water and gas/heat meters, micro generation devices, or act as a gateway for the smart home) and build a so called advanced metering infrastructure (AMI) [1]. Another important task of smart meters is load management as they are able to trigger relays to connect/disconnect specific loads. Smart meters can be remotely controlled by utilities, e.g. the maximum allowed consumption can be restricted or the electricity of a connected customer can be switched off.

Repeaters are regular smart meters that act as gateways for other smart meters. Each smart meter is registered at one concentrator, which controls the meter and to which the meter is reporting its data. In case a smart meter cannot connect directly to a concentrator (due to distance, noises, interference signals) it can use another smart meter as repeater. This can be repeated multiple times until a concentrator is reached. This characteristic organizes the communication topology of the smart grid. However, the topology is dynamic and changes over time, e.g. if a repeater fails, a physical cable connection is broken, or noises disturb the communication. In such cases smart meters dynamically reorganize the topology by reconnecting to other smart meters (see section II-C).

Water meters are used to measure water consumption of customers. Usually, they are less powerful devices than smart meters, have only limited functionalities, (e.g. regarding flow control compared to load management on an electrical smart meter). In Luxembourg, water meters are not directly

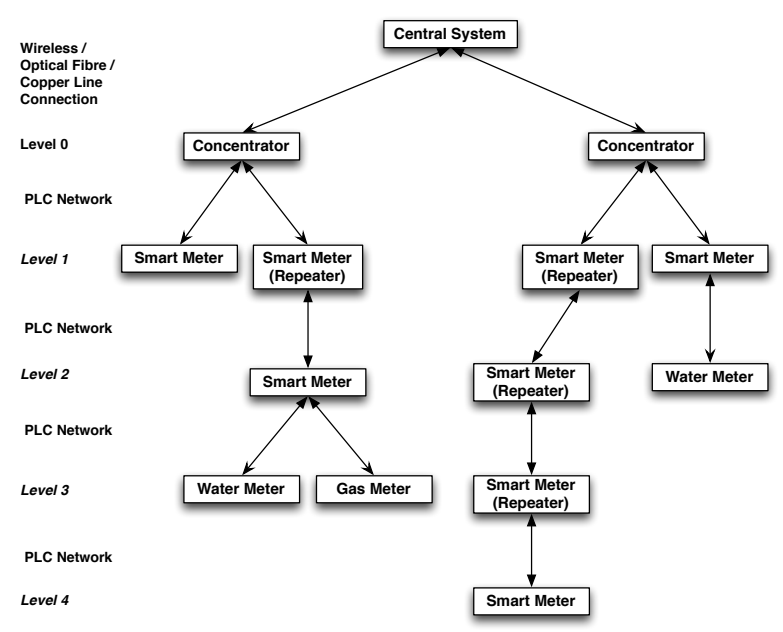

Fig. 1. Schematic representation of the smart grid communication topology

connected to a data concentrator but connected (and controlled) via a smart meter. The communication between water meters and the electricity smart meters is encrypted. In other countries water meters are directly connected to a concentrator via wireless communication. In Luxembourg this solution is not considered for now but technically it would be feasible.

Gas or heat meters are used to measure gas consumption of customers. Similar to water meters they are usually limited devices and are connected (and controlled) via a smart meter.

Data concentrators collect and store (for several months) consumption data from a number of associated meters. In regular intervals (typically several times a day, in some settings immediately) they send this data (usually via IP connections) to a central control system. During the pilot phase in the Luxembourg deployment the data are send via GPRS connections [10] but in future other technologies such as optical fibre and copper lines will be used as well. All smart meters connected (either directly or via repeaters) to a concentrator are controlled by it. Therefore, concentrators have the ability to send commands, like requesting consumption data or to shut down electricity. Physically, data concentrators in Luxembourg are located at power substations. In case of bigger housing complexes a concentrator can also be located directly in the housing complex itself. Concentrators are configured by a central system but work afterwards autonomously. Some concentrator implementations read meters strictly sequential (asking a meter, waiting of the answer/timeout, asking next meter, waiting, ...) while others are able to read meters in parallel (asking a meter and continue with asking the next one without waiting for an answer). Concentrators divide the smart grid communication topology into smaller, autonomous regions, which are not directly connected to each other, enabling the distributed control ability of the smart grid [1].

Central system: concentrators send their data to a central system where all data are stored, aggregated and analyzed. Because of legal regulations these data must be deleted in regular intervals (e.g. cannot be stored longer than $x$ month).

Fig. 1 shows schematically how the smart grid communication topology in Luxembourg is organized. As can be 
seen in the figure, the topology is organized as a tree. Each concentrator is the root node of a subtree. The individual subtrees are connected (e.g. through wireless connections, optical fibre, copper line) to the central system. Entities inside a concentrator subtree are connected through a PLC network. In this way, each data concentrator controls one region of connected smart meters. In average between 50 and 150 smart meters are connected to one concentrator. Smart meters can be either directly connected to a concentrator or, in case the signal is too weak, connected to a concentrator via one or more repeaters. Apart from the fact that repeaters are used as gateways by other smart meters to reach a concentrator, they are regular smart meter devices. In fact, each smart meter is able to be used as a repeater. At a time $t$ each smart meter can be connected (directly or via a repeater) to at most one concentrator. Similar, at a time $t$, a smart meter, which is not directly connected to a concentrator, can use at most one other smart meter as repeater. This characteristic leads to the tree structure of the communication topology.

We analyzed three different regions (each with one data concentrator) in Luxembourg in detail. Therefore, we evaluated - together with our industrial partner Creos Luxembourg S.A. - a set of real-world data that contains: the above described communication topology of smart meters, concentrators, and the central system; how the topology changes over time; complete consumption and production data over a period of several days (one value each 15 minutes); GPS locations of entities; location and characteristics of physical electricity cables used for the PLC network; the success rates of meter readings (over a period of time); and duration of meter readings. We evaluate this data and present the key characteristics of the outcome in the following sections.

\section{B. Topology Characteristics}

The evaluation has shown that in the three examined regions between 55 and 102 smart meters are connected to a concentrator. This makes an average of $N=75.6$ smart meters per concentrator. We found that between 9 and 27 smart meters per region act as repeaters. This is in average 20.3 smart meters per region. Furthermore, we found that the path length from a smart meter to a concentrator in the examined regions is at least 1 and at most 7 . The path length from source (smart meter) to destination (concentrator) is usually called the number of hops [11] form source to destination. For example, in fig. 1 all nodes on level 1 have a path length (number of hops) to the concentrator of 1 , all nodes on level 2 a path length of 2 , and so forth. The average number of hops in the three regions, over a timeframe of 24 hours, is 2.6. Besides the number of hops we also analyzed the actual physical distance $d i$ from the smart meters to data concentrators. Our study showed that this distance is between 7 and 501 meters. In average (over all three regions) the distance between a smart meter and a concentrator is 317.6 meters. Next let's consider the degree of a node in the communication topology tree. In this context with degree of a node we mean the number of edges (communication connections) going in or coming out of a node. As can be seen in fig. 1 a concentrator has one connection (wireless, optical fibre, copper line) to the central system. In addition, each concentrator has one connection to each directly connected smart meter. This leads to a degree of $N+1$ per concentrator, where $N$ again is the number
TABLE I. COMPARISON OF THREE REGIONS

\begin{tabular}{|l|l|l|l|}
\hline & Region 1 & Region 2 & Region 3 \\
\hline \hline Nodes & 102 & 70 & 55 \\
\hline Repeaters & 27 & 25 & 9 \\
\hline $\begin{array}{l}\text { Path length } \\
\text { (min / max / avg) }\end{array}$ & $1 / 7 / 3.0$ & $1 / 5 / 2.3$ & $1 / 3 / 1.9$ \\
\hline $\begin{array}{l}\text { Distance (meter) } \\
\text { (min / max / avg) }\end{array}$ & $10 / 501 / 256.5$ & $40 / 479 / 219.1$ & $7 / 466 / 212.3$ \\
\hline $\begin{array}{l}\text { Meter reading } \\
\text { success rate (\%) } \\
\text { (min / max / avg) }\end{array}$ & $89.0 / 91.2 / 90.1$ & $78.5 / 82.6 / 81.2$ & $74.8 / 80.8 / 78.0$ \\
\hline $\begin{array}{l}\text { Duration to } \\
\text { read (seconds) } \\
\text { (min / max / avg) }\end{array}$ & $1.96 / 8.31 / 4.52$ & $2.09 / 6.47 / 3.63$ & $3.64 / 8.02 / 5.62$ \\
\hline
\end{tabular}

of directly connected smart meters. We evaluated a degree between 10 and 28 for concentrators. The average degree of a concentrator in the three examined regions is 19.7. Each smart meter that is not acting as a repeater is either directly connected to a concentrator or to another smart meter (repeater). In addition, a smart meter can have up to two connections, one to a water meter and another one to a gas or heat meter. This leads to a degree between 1 (no water and gas/heat meter, not acting as a repeater, connected to a concentrator or another repeater) and 3. We have no information about how many smart meters are connected to water and/or gas/heat meters so we cannot evaluate the average degree of a smart meter. A smart meter that acts as a repeater for other smart meters has, in addition to the connections of a regular smart meter, one connection to each of them. Therefore, the degree of a repeater is between 2 (no water and gas/heat meter, acting as a repeater, connected to a concentrator or another repeater) and $N$. Due to the lack of information about how many smart meters (repeaters) are connected to water and/or gas/heat meters we cannot evaluate the exact average degree of a repeater. Ignoring water and gas/heat meters we evaluated a degree between 2 and 11 with an average of 3.75 . For water meters and gas/heat meters the degree is straightforward 1 . Another criteria we analyzed is the success rate of meter reading. Due to electrical noise and disturbances on the PLC network, attempts to remotely read a meter are not always successful. We found that the success rate of meter reading is comparatively high. We analyzed the meter reading in a range of seven days and found that the meter reading success rate was between $74.8 \%$ and $91.2 \%$. The average success rate over seven days was $83.3 \%$. This means that in average every fifth reading attempt fails. Another characteristic we investigated is the duration to read meters (time until the concentrator receives an answer from the meter). The fastest reply in the investigated time window was 1.96 seconds and the slowest 8.31 seconds. In average (over all three regions) a meter reading takes 4.51 seconds.

The evaluation of the data shows that the communication topology of the smart gird is divided into relatively small subtrees with a manageable number of nodes, hops, a small degree level for nodes, and only small physical distances between smart meters and concentrators. Table I compares all these data for the three regions.

\section{Evolution over Time}

As discussed, the communication topology is not fixed but continuously evolves. For example, due to noises or defects on cables, or not reachable meters, smart meters can use other smart meters as repeaters, which leads to a change of the 
TABLE II. LUXEMBOURG SMART GRID CHARACTERISTICS

\begin{tabular}{|c|c|}
\hline Characteristic & Value \\
\hline Topology structure & Tree; one subtree for each concentrator \\
\hline Nodes $N$ per region & min: 30 / max: 102 / avg: 67.3 \\
\hline Repeaters per region $R$ & min: 9/ max: 27 / avg: 20.3 \\
\hline Percentage of pepeaters & min: $30 \% /$ max: $26.5 \% /$ avg: $30.2 \%$ \\
\hline Number of hops $h$ & min: 1/ max: 7 / avg: 2.6 \\
\hline $\begin{array}{l}\text { Degree } d \\
\text { - for a smart meter } \\
\text { - for a repeater } \\
\text { - for a water / gas / heat meter } \\
\text { - for a concentrator } \\
\text { - for the central system }\end{array}$ & $\begin{array}{l}\text { up to } 3 \\
\text { min: } 2 \text { / max: } 11 \text { / avg: } 3.75 \\
\text { up to } 1 \\
\text { min: } 10 \text { / max: } 28 \text { / avg: } 21.3 \\
\text { number of concentrators: } 3\end{array}$ \\
\hline Distance $d i$ & min: 7 / max: 501 / avg: 317.6 \\
\hline Topology changes per hour $c$ & min: 0 / max: 273 / avg: 31 \\
\hline Meter reading success rate $(\%) r$ & min: 74.8 / max: 91.2 / avg: 83.3 \\
\hline Duration to read (seconds) & min: 1.96 / max: $8.31 /$ avg: 4.51 \\
\hline
\end{tabular}

communication topology. We monitored all status changes of smart meters (including those acting as repeaters) over a period of 48 hours. For the evaluation of the data we restricted to those status changes, which indicate a registering of a smart meter. This roughly reflects the magnitude of topology changes. We discovered that such changes occur comparatively frequent: 3485, 414, respectivly 692 times in the investigated time period. This corresponds to an average of 1530 changes per 48 hours or more than 31 changes each hour. Fig. 2 shows for each region the course of topology changes over the investigated time window of 48 hours.

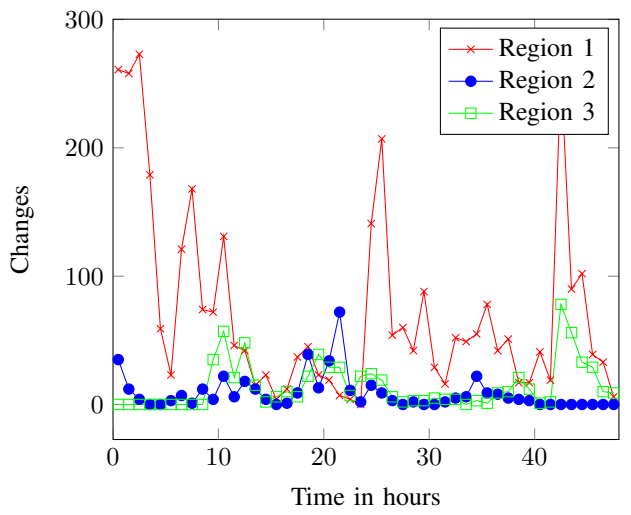

Fig. 2. Topology change rate

\section{Summary}

Table II summarizes the above discussed main characteristics of the communication topology of the Luxembourg smart grid. The table shows the smallest (min), largest (max), and average (avg) value found for each characteristic. Fig. 3 shows a snapshot of the communication topology of one of the considered regions in Luxembourg. The figure is based on real data and contains smart meters, repeaters, and one data concentrator. The connections between the above mentioned entities are logical connections not physical PLC network connections. This means that they represent the current structure of the topology, e.g. which smart meter is connected to which repeater or concentrator. This example shows the size and communication structure of a typical region in Luxembourg. An interesting aspect, which can be derived from this topology model is that there is one repeater connecting two parts of the region (single connection point). This connection is critical

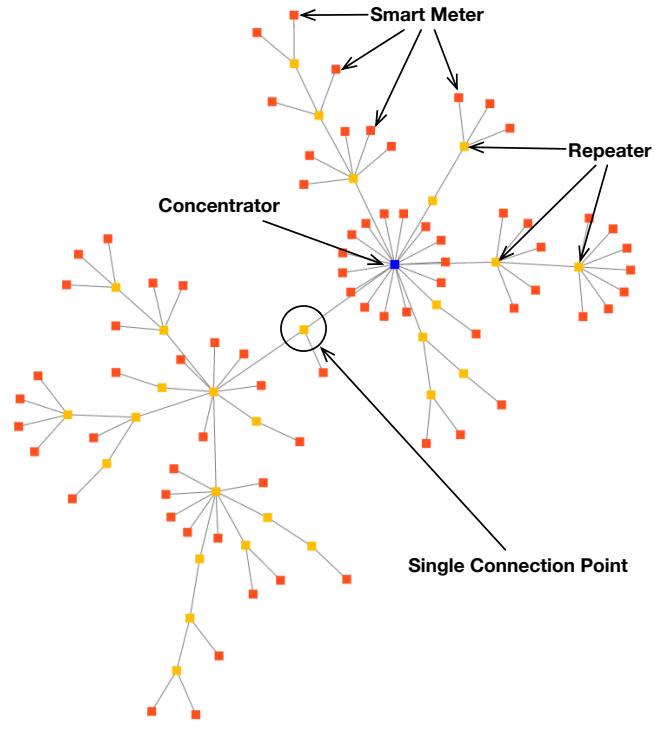

Fig. 3. Example topology of one of the considered regions in Luxembourg

since the complete left subtree would be no longer reachable if this single repeater is broken. This shows how the analyzis of a topology model can help to improve the actual topology. For example, in this case the protocol how meters connect to other meters could be optimized in a way that such singe connection points are avoided or at least minimized.

\section{SMART GRID COMMUNICATION TOPOLOGY GENERATOR}

In this section we describe our smart grid communication topology generator. This generator is able to create random but realistic smart grid communication topologies based on the results of our smart grid analysis. The complete source code of the generator can be found on github ${ }^{2}$.

\section{A. Smart Grid Meta-Model}

We first define an abstract smart grid meta-model based on the findings of our analysis from section II. This metamodel can be seen as a generalization of the Luxembourg smart grid communication topology. Fig. 4 shows an excerpt of it. The meta-model is the formal description of the topology characteristics. It describes the concepts required to model an abstraction of a PLC smart grid communication topology. It contains the topological entities as well as the physical communication structure. As can be seen in the figure, an entity can register itself by another entity. This concept allows to model that smart meters can connect to concentrators as well as that smart meters use other smart meters as repeaters. In addition the meta-model contains the physical location of each entity as well as the physical start and end points of wired communication medias. This meta-model is central for our generator. It defines all concepts and the structural definition of all possible topologies. We use it as a template definition to which all generated random topologies our generator produces must conform. This ensures their structural correctness.

\footnotetext{
${ }^{2}$ https://github.com/thomashartmann/smartgrid-topology-generator
} 


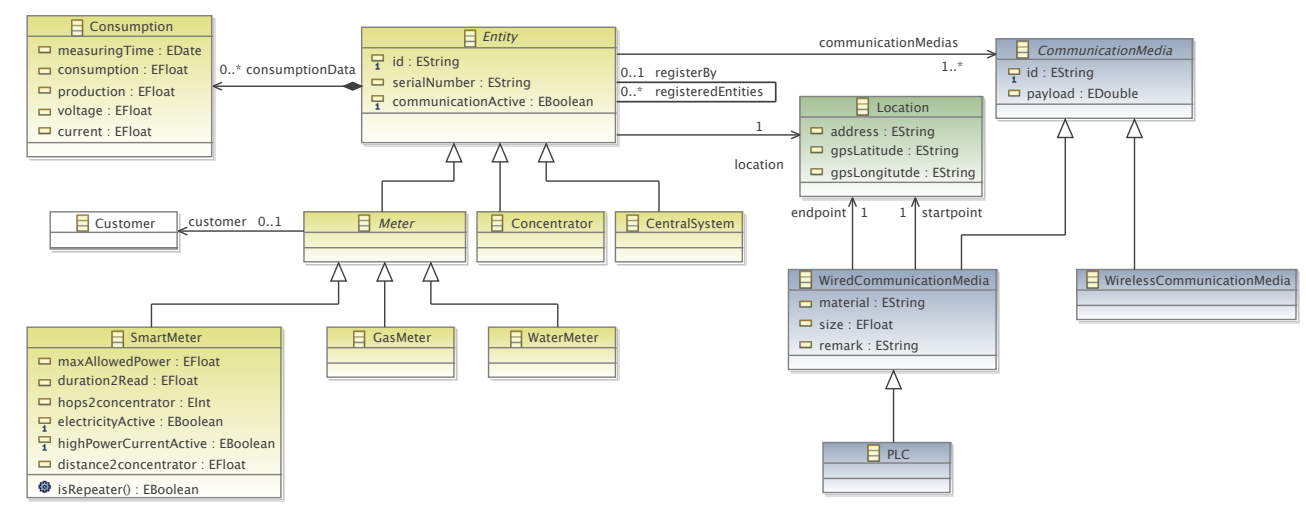

Fig. 4. Smart grid meta-model

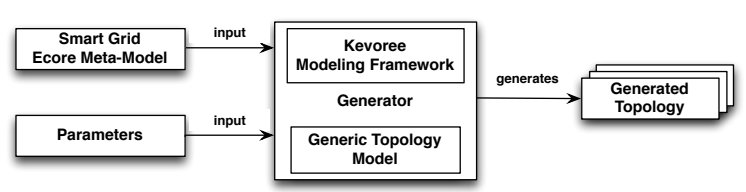

Fig. 5. Generator Architecture

\section{B. Generator Architecture Overview}

We use an Ecore representation of our meta-model to generate smart grid communication topologies. Ecore is the meta-model of the Eclipse Modeling Framework (EMF) [12]. It allows to describe models with constructs such as packages, classes, associations, inheritance, and attributes. Ecore is similar (with few differences) to OMG's Essential Meta Object Facility (EMOF) and is regarded as its reference implementation. We use Ecore because it is the de-facto standard for defining models, so that there exists a lot of tools supporting Ecore meta-models. We build our generator on top of the Kevoree Modeling Framework (KMF) ${ }^{3}$ [13]. KMF can be seen as an alternative, lightweight implementation of EMF, which comes without dependencies to the Eclipse platform. It supports the standard XMI format for compatibility with other modeling frameworks and tools, especially EMF. Like EMF, KMF allows to generate a set of classes (e.g. in Java) from a Ecore meta-model. Based on our analysis of typical smart grid communication topologies we use these classes in our generator to create random but realistic topologies, which conform to the meta-model. We extract average metrics from our analysis of real data (such as distance, geographical distribution) to feed the generator to create random topologies mapping to these metrics. The results of the topology analysis are implemented as generation rules. For example, such a rule is that each smart meter is connected (directly or via repeaters) to at most one concentrator. Fig. 5 shows an overview of the architecture we use to implement the generator. The generator can be parameterized to control properties of the output topologies. By default these parameters are predefined with the values derived from our analysis of the Luxembourg smart grid communication topology. Table III lists all possible properties. The generated topologies can be exported in XMI

\footnotetext{
${ }^{3}$ http://kevoree.org/kmf/
}

TABLE III. GENERATOR PARAMETERS

\begin{tabular}{|l|l|}
\hline Property & Description \\
\hline \hline NB_CON & Number of concentrators (number of subtrees). \\
\hline MIN_NB_SM & Min number of smart meters per concentrator. \\
\hline MAX_NB_SM & Max number of smart meters per concentrators. \\
\hline MIN_DISTANCE & Min distance from smart meters to concentrator. \\
\hline MAX_DISTANCE & Max distance from smart meters to concentrator. \\
\hline MIN_NB_HOPS & Min number of hops from smart meter to concentrator. \\
\hline MAX_NB_HOPS & Max number of hops from smart meter to concentrator. \\
\hline PERCENTAGE_WM & Percentage of smart meters controlling a water meter. \\
\hline PERCENTAGE_GM & Percentage of smart meters controlling a gas meter. \\
\hline
\end{tabular}

and JSON and therefore be easily used by third-party tools. The output topologies represent random topologies at one point of time. This means time-evolution is not part of generated topologies nor of the meta-model. However, we are working on modeling and reasoning strategies to integrate time-evolution into context models [14] and plan to use this approach to extend our generator, so that time-evolution can be integrated into the generated topologies.

\section{RELATED WORK}

Most existing work concentrate on the transmission domain of the power grid. A typical transmission system forms a generic graph and can span over a whole country or even a continent. The properties of this graph are studied from a network point (clustering coefficient, node degree distribution) for isolating critical components, e.g. transmission lines or power plants. In [15] the authors study topological vulnerabilities of transmission systems of several European countries, while [16] focus on US transmission systems. The major similarity among these papers is that the power grid topology exhibits characteristics, which differ from random graphs, preferential attachment and small-world graphs. The purely topological approach may sometimes lead to inaccurate conclusions if the domain specificities are ignored. Several authors have worked in this direction by introducing new metrics related to the power flow [17] or the resistive distance in the network [18]. Pagani et al. provide a survey [19] on a network approach to power grid topology analysis. The distribution network having a tree structure and interconnecting a much lower number of consumers, presents a lesser interest for the power engineering community. However, the interest in these topologies is increasing with the emergence of dynamic network reconfiguration, mainly for increased energy efficiency 
and renewable energy integration. The closest to our study is [20], where the authors analyse the distribution network in the Netherlands, with network characteristics comparable to the ones we present in our paper. Smart grid communication networks over PLC have become a center point of interest in past years. The need of topology generation suitable for network simulators is therefore of great importance.

\section{FUTURE WORK}

In future work we will integrate this topology model into a simulator. We are working on a tool for reactive security [21]. This tool uses the topology model presented in this paper to simulate impacts of attacks and failures and, in addition, behaviour definition to simulate and evaluate possible countermeasures. Another area of interest is, how we can improve routing algorithms. Based on the topology model combined with real-time information of the grid status (e.g. broken cables, defect repeaters) we plan to dynamically improve and adapt routing strategies. Currently time-evolution of topologies is not part of the generated topologies. Instead, they only reflect the topology state at one point in time. We are working on a modeling and reasoning approach to integrate timeevolution directly into context models [14]. Finally, we plan to investigate the use of probability distributions instead of $\min / \max$ values for generated topologies.

\section{CONCLUSION}

The transition of today's centralized electricity grid into a distributed, self-adaptive, and self-healing smart grid is still in an early stage. Until the smart grid vision turns into reality much work still needs to be done by researchers, engineers, and industrial professionals. One backbone of this effort is the facilitation of ICT to allow two-way communication and distributed control of devices. The underlying communication topology is a major characteristic of a smart grid. Many tasks, such as analyzing, simulating, designing, and comparing smart grid infrastructures but also optimizing routing algorithms, and predicating impacts of events and failures rely on realistic abstractions of smart grid communication topologies. However, since smart grids are emerging infrastructures that are still in a research and test phase it is difficult to get access to realistic communication topologies. Through the collaboration with our industrial partner, Creos Luxembourg S.A., we had the opportunity to analyze the communication topology of the smart grid currently deployed in Luxembourg. In this paper we presented and discussed main static as well as dynamic characteristics of this communication topology. Finally, we presented a generator that is able to create random but realistic smart grid communication topologies based on the characteristics found by our analyzes of the Luxembourg smart grid topology. The generator can be parameterized to create topologies of different sizes and with different properties. These topologies can be used for analyzing, simulating, and designing smart grid infrastructures. For example, by using topology models to simulate impacts of attacks and failures new opportunities to analyze safety aspects of smart grids can be created.

\section{ACKNOWLEDGMENT}

The authors would like to thank Yves Reckinger and Robert Graglia form Creos Luxembourg S.A. for their support.

\section{REFERENCES}

[1] H. Farhangi, "The path of the smart grid," Power and Energy Magazine IEEE, vol. 8, no. 1, pp. 18-28, January 2010.

[2] P. Samadi, H. Mohsenian-Rad, V. Wong, and R. Schober, "Real-time pricing for demand response based on stochastic approximation," Smart Grid, IEEE Trans. on, vol. 5, no. 2, pp. 789-798, March 2014.

[3] J. Rothenberg, L. E. Widman, K. A. Loparo, and N. R. Nielsen, "The nature of modeling," in in Artificial Intelligence, Simulation and Modeling. John Wiley Sons, 1989, pp. 75-92.

[4] Z. Wang, A. Scaglione, and R. Thomas, "Generating statistically correct random topologies for testing smart grid communication and control networks," Smart Grid, IEEE Trans. on, pp. 28-39, 2010.

[5] A. J. Holmgren, "Using graph models to analyze the vulnerability of electric power networks," Risk Anal., vol. 26, no. 4, pp. 955-969, 2006.

[6] S. Galli, A. Scaglione, and Z. Wang, "For the grid and through the grid: The role of power line communications in the smart grid," Proc. of the IEEE, vol. 99, no. 6, pp. 998-1027, June 2011.

[7] A. Aruzuaga, I. Berganza, A. Sendin, M. Sharma, and B. Varadarajan, "Prime interoperability tests and results from field," in Smart Grid Communications, 2010 1st IEEE Int. Conf. on, 2010.

[8] K. Razazian, M. Umari, A. Kamalizad, V. Loginov, and M. Navid, "G3-plc specification for powerline communication: Overview, system simulation and field trial results," in Power Line Communications and Its Applications, IEEE Int. Symp.um on, March 2010, pp. 313-318.

[9] S. Ramseler, M. Arzberger, and A. Hauser, "Mv and lv powerline communications: new proposed iec standards," in Transmission and Distribution Conference, IEEE, vol. 1, Apr 1999, pp. 235-239.

[10] P. Parikh, M. Kanabar, and T. Sidhu, "Opportunities and challenges of wireless communication technologies for smart grid applications," in Power and Energy Society General Meeting, IEEE, July 2010, pp. 1-7.

[11] M. Biagi and L. Lampe, "Location assisted routing techniques for power line communication in smart grids," in Smart Grid Communications (SmartGridComm), 1st IEEE Int. Conf. on, Oct 2010, pp. 274-278.

[12] F. Budinsky, D. Steinberg, and R. Ellersick, Eclipse Modeling Framework : A Developer's Guide, 2003.

[13] F. Fouquet, G. Nain, B. Morin, E. Daubert, O. Barais, N. Plouzeau, and J. Jézéquel, "An eclipse modelling framework alternative to meet the models@ runtime requirements." in MoDELS, 2012.

[14] T. Hartmann, F. Fouquet, G. Nain, B. Morin, J. Klein, and Y. Le Traon, "Reasoning at runtime using time-distorted contexts: A models@run.time based approach," in Proc. of the 26th Int. Conf. on Software Engineering and Knowledge Engineering, 2014, pp. 586-591.

[15] M. Rosas-Casals, S. Valverde, and R. V. Sol, "Topological vulnerability of the european power grid under errors and attacks." I. J. Bifurcation and Chaos, vol. 17, no. 7, pp. 2465-2475, 2007.

[16] P. Hines, S. Blumsack, E. Cotilla Sanchez, and C. Barrows, "The topological and electrical structure of power grids," in System Sciences (HICSS), 43rd Hawaii International Conference on, Jan 2010, pp. 1-10.

[17] S. Arianos, E. Bompard, A. Carbone, and F. Xue, "Power grid vulnerability: A complex network approach," Chaos: An Interdisciplinary Journal of Nonlinear Science, vol. 19, no. 1, 2009.

[18] E. Cotilla-Sanchez, P. Hines, C. Barrows, and S. Blumsack, "Comparing the topological and electrical structure of the north american electric power infrastructure," Systems Journal, IEEE, vol. 6, no. 4, pp. 616626, Dec 2012.

[19] G. A. Pagani and M. Aiello, "The power gridas a complex network: A survey," Physica A: Statistical Mechanics and its Applications, vol. 392, no. 11, pp. 2688-2700, 2013.

[20] — - "Towards decentralized trading: A topological investigation of the dutch medium and low voltage grids," CoRR, vol. abs/1101.1118, 2011.

[21] T. Hartmann, F. Fouquet, J. Klein, G. Nain, and Y. Le Traon, "Reactive Security for Smart Grids using Models@run.time-Based Simulation and Reasoning," in Second Open EIT ICT Labs Workshop on Smart Grid Security, Feb 2014. 